\title{
Hombre de 77 años con dolor lumbar: reporte de un caso
}

\author{
Estefany L. Tamayo-Pérez ${ }^{*}$, Sergio A. Giraldo-Serrano ${ }^{1}$, Laura S. Nasiff-Cabrales ${ }^{1}$ y \\ María A. Muñoz-Rubiano² \\ ${ }^{1}$ Servicios Médicos de Emergencia y Trauma, ISMET, Facultad de Emergenciología, Universidad del Rosario, Fundación Santa Fé de Bogotá; \\ ${ }^{2}$ Departamento de Epidemiología, Hospital de Kennedy. Bogotá D.C., Colombia
}

\section{Resumen}

Introducción: El dolor lumbar es un síntoma inespecífico, un motivo de consulta frecuente en urgencias acompañado de múltiples síndromes de diferentes grados de severidad. El objetivo es dirigir el diagnóstico hacia una etiología no musculoesquelética. Presentación de caso: Hombre de 77 años, consulta por dolor lumbar de tres días irradiado a miembro inferior derecho, al ingreso con taquipnea, disnea y requerimiento de oxígeno con máscara de no reinhalación. Al examen físico se encuentra distensión abdominal; gases arteriales con acidosis metabólica e hiperlactatemia. Se sospecha enfermedad aórtica y/o isquemia mesentérica; siendo la angio-tomografía toraco-abdominal negativa para estas, se llega a un diagnóstico. Conclusión: La espondilodiscitis es una entidad poco frecuente, con un aumento de la incidencia en los últimos años, se presenta habitualmente con dolor lumbar y fiebre, algunos con síntomas neurológicos como una de las complicaciones más severas, por lo que su abordaje es un reto diagnóstico.

Palabras clave: Dolor lumbar. Espondilodiscitis. Sepsis. Enfermedad aórtica.

\section{7-year-old man with low back pain: case report}

\begin{abstract}
Introduction: Lumbar pain is an unspecified symptom and a chief complaint in the emergency room secondary to different etiologies of complexity degree. The aim is to identify a non-musculoskeletal etiology. Case report: 77-year-old patient arrives to the emergency room with 3 days of onset of lumbar pain, it irradiated towards the right lower extremity. Upon admission patient is tachypneic, dyspneic and requiring oxygen with a non- rebreathing mask. Upon physical examination abdominal distension was found, arterial gases with metabolic acidosis and hyperlactatemia, diagnostic impression was aortic disease or Mesenteric ischemia, however Angio-CT was negative, a diagnosis has been reached. Conclusion: Spondylodiscitis is a rare entity, with an increase in its incidence in the recent years, chief complaint is lumbar pain and fever, in more severe cases neurological symptoms are associated, diagnosis of this entity in the emergency room is a challenge.
\end{abstract}

Keywords: Low back pain. Spondylodiscitis. Sepsis. Aortic disease.

\section{Correspondencia:}

*Estefany L. Tamayo-Pérez

E-mail: estefany.tamayo@urosario.edu.co
Fecha de recepción: 17-04-2021

Fecha de aceptación: 30-08-2021 DOI: 10.24875/REIE.21000039
Disponible en internet: 10-11-2021 Rev Educ Investig Emer. 2022;4(1):40-44 www.medicinadeemergencias.com bajo la licencia CC BY-NC-ND (http://creativecommons.org/licenses/by-nc-nd/4.0/). 


\section{Descripción del caso}

Hombre de 77 años en postoperatorio mediato de drenaje de hematoma subdural. Ingresa a urgencias por tres días de dolor urente lumbar; intensidad 10/10 según escala análoga verbal, irradiado a miembro inferior derecho, exacerbado cuatro horas previas a la consulta, asociado con disnea y dolor abdominal. Ingresó taquipneico, con cianosis central, distensión abdominal y radiculopatía dorsolumbar. Gases arteriales con acidosis metabólica, hiperlactatemia y trastorno moderado de oxigenación.

Por falla ventilatoria tipo I vs. IV, dolor lumbar con «banderas rojas»y distensión abdominal. Se sospechó síndrome aórtico agudo, isquemia mesentérica y/o tromboembolia pulmonar, sin embargo, las angio-tomografías de tórax y abdomen descartan estas enfermedades. Laboratorios con leucocitosis, neutrofilia, trombocitopenia, lesión renal aguda, hiperbilirrubinemia, transaminitis, cetonuria y glucemia normal. Adicionalmente se considera cetoacidosis diabética euglucémica por uso previo de inhibidores del cotransportador sodio- glucosa tipo 2. SOFA (Sequential Organ Failure Assessment) score de ingreso 8 puntos, por posible infección abdominal se inició piperacilina tazobactam e infusión de insulina cristalina.

En la unidad de cuidados intensivos se realizó resonancia magnética nuclear de columna (Fig. 1), con espondilodiscitis L4-L5, absceso paravertebral posterior y en psoas derecho, se llevó a drenaje, cultivo con Klebsiella pneumoniae, se ajustó antibiótico intrahospitalario con ertapenem y continuidad ambulatoria con ampicilina sulbactam durante 50 días.

\section{Introducción}

La lumbalgia es un síntoma inespecífico relacionado con múltiples enfermedades de diferente severidad ${ }^{1}$. Tiene incidencia anual del $5 \%$ y afecta al $90 \%$ de la población en algún momento de su vida ${ }^{2}$. Usualmente es benigno, sin embargo, se deben excluir etiologías no musculoesqueléticas ${ }^{1}$.

En una revisión sistemática de Maselli y Testa se identificaron signos y síntomas para detectar patologías graves ${ }^{3}$. Los principales predictores son la fiebre (con sensibilidad del 97-99\%), diaforesis, lumbalgia nocturna, pérdida de peso y síntomas neurológicos que sugieran compromiso sistémico ${ }^{3}$.

Deyo y Weinstein en 2001 proponen tres preguntas para descartar patologías de origen no musculoesquelético ${ }^{4}$. 1) ¿El dolor es de origen abdominal o retroperitoneal? ${ }^{4,5}$ 2) ¿Hay compromiso neurológico?,

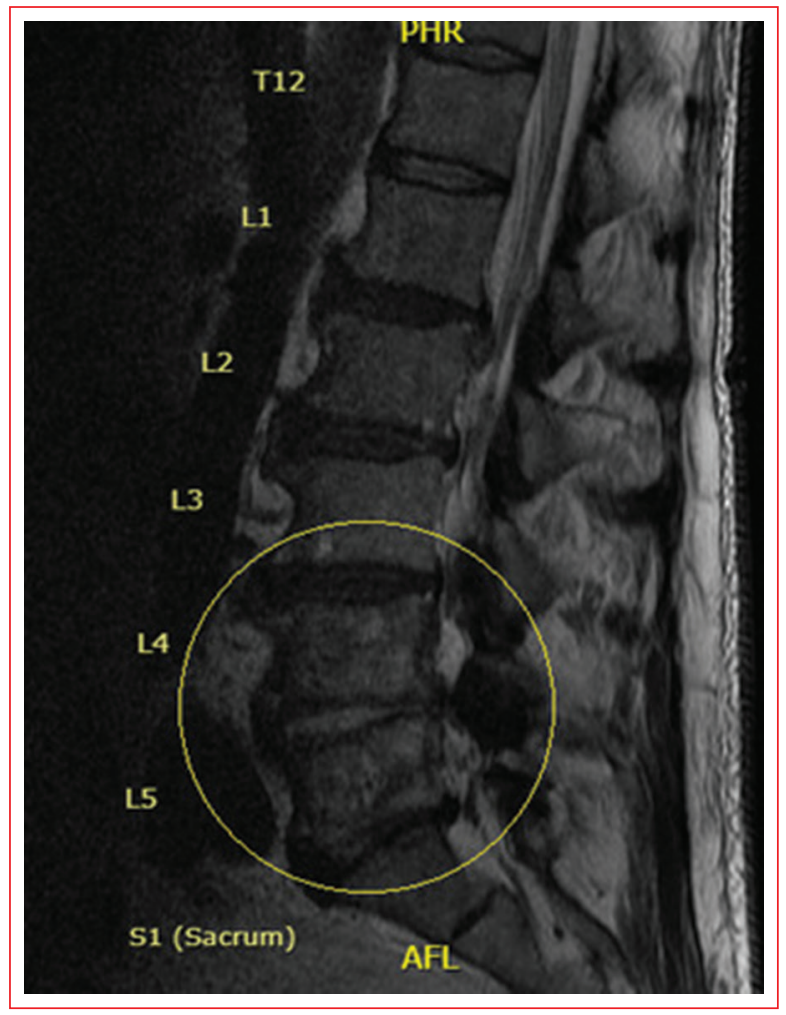

Figura 1. Compromiso inflamatorio paraespinal anterior y posterior, con pequeña colección paraespinal posterior derecha en L4-L5 y realce anormal con el medio de contraste que involucra el espacio epidural, con una pequeña colección sublaminar derecha en S1.

sospechar discapacidad grave, y 3) ¿La patología es degenerativa?, sospechar hernias discales, lesiones musculares o ligamentosas ${ }^{4,5}$. En este estudio la infección estuvo presente en el $0.01 \%$ de los pacientes ${ }^{4,5}$.

La espondilodiscitis es la inflamación del disco y el cuerpo vertebral ${ }^{1,2,4}$. El aumento en la incidencia es el reflejo de una mayor sospecha y expectativa de vida ${ }^{6,7}$. Generalmente es crónico, su diagnóstico es difícil y retrasar el tratamiento aumenta la morbimortalidad 7 . Se realiza este reporte de caso por la inusualidad de la enfermedad y la severidad.

\section{Epidemiología}

Se estima que la espondilodiscitis equivale al $2.7 \%$ de las osteomielitis, su incidencia anual en países occidentales es de 0.5 a 2.5 por 100,000 habitantes por año6; su distribución es binomial, con un primer pico en jóvenes y el segundo en la sexta década de la vida, su incidencia en menores de 20 años es de 0.3 y en mayores de 70 años de 6.5 por 100,000 habitantes, respectivamente ${ }^{8,9}$. 
Los factores predisponentes son el uso crónico de corticosteroides o inmunosupresores, drogas intravenosas, enfermedad renal, neoplasias y procedimientos quirúrgicos con una incidencia que varía del 0.5 al $18.8 \% \%^{6,7}$. Las complicaciones más frecuentes son los abscesos paravertebrales en el $26 \%$, epidurales en el $17 \%$ y compromiso del disco intervertebral en un $5 \%$, con una recaída del $8 \%$ y mortalidad del 6\% $\%^{8-10}$.

\section{Fisiopatología}

La irrigación del disco intervertebral es limitada e indirecta, los vasos entran hasta el anillo fibroso, siendo los cuerpos vertebrales avasculares, su irrigación va a depender del flujo sanguíneo, originado cerca al ligamento anterior longitudinal $1^{9,11-13}$. El microorganismo atraviesa la placa vertebral cartilaginosa, recorre la superficie del disco, infecta la metáfisis vertebral adyacente y llega al espacio discal ${ }^{9,11-13}$. Como resultado hay oclusión del flujo sanguíneo, creando necrosis avascular, infarto óseo y/o espondilodiscitis ${ }^{9,11-13}$.

Existen tres vías de colonización bacteriana; la primera es por diseminación hematógena ${ }^{9,11-13}$, la segunda es la inoculación directa por procedimientos invasivos y la tercera es la colonización por continuidad de tejidos infectados adyacentes ${ }^{8,14}$. McHenry, et al., en un estudio retrospectivo en Cleveland de 253 pacientes con osteomielitis vertebral, reportan bacteriemia en el $62 \%$ de los pacientes, de estos el $51 \%$ tenían origen urinario, de tejidos blandos, punciones vasculares, endocarditis, bursitis y/o artritis ${ }^{15}$.

La infección asociada a cirugía está documentada en el $33 \%$ de los casos, por inoculación directa relacionada con otros procedimientos invasivos como discografías, cateterización epidural, bloqueos, aortografía translumbar y punción lumbar, entre otros.

Por último, en un 3\% la infección por contigüidad, como en abscesos adyacentes y/o úlceras de presión infectadas $^{14,15}$.

La causa principal es por diseminación hematógena, esto exige la búsqueda activa de un foco oculto, interrogando antecedentes quirúrgicos, uso de drogas intravenosas, lesiones cutáneas, síntomas urinarios o uso previo de antimicrobianos que condicionen resistencia bacteriana ${ }^{8}$.

En infecciones de transmisión hematógena, el microorganismo más común es Staphylococcus aureus, responsable del $30-80 \%$ de los casos, seguido de $\mathrm{E}$. coli8,14. En un estudio observacional realizado en Dinamarca de 1980 a 1990, incluyeron 8,739 pacientes con bacteriemia por $S$. aureus, 309 tuvieron osteomielitis,

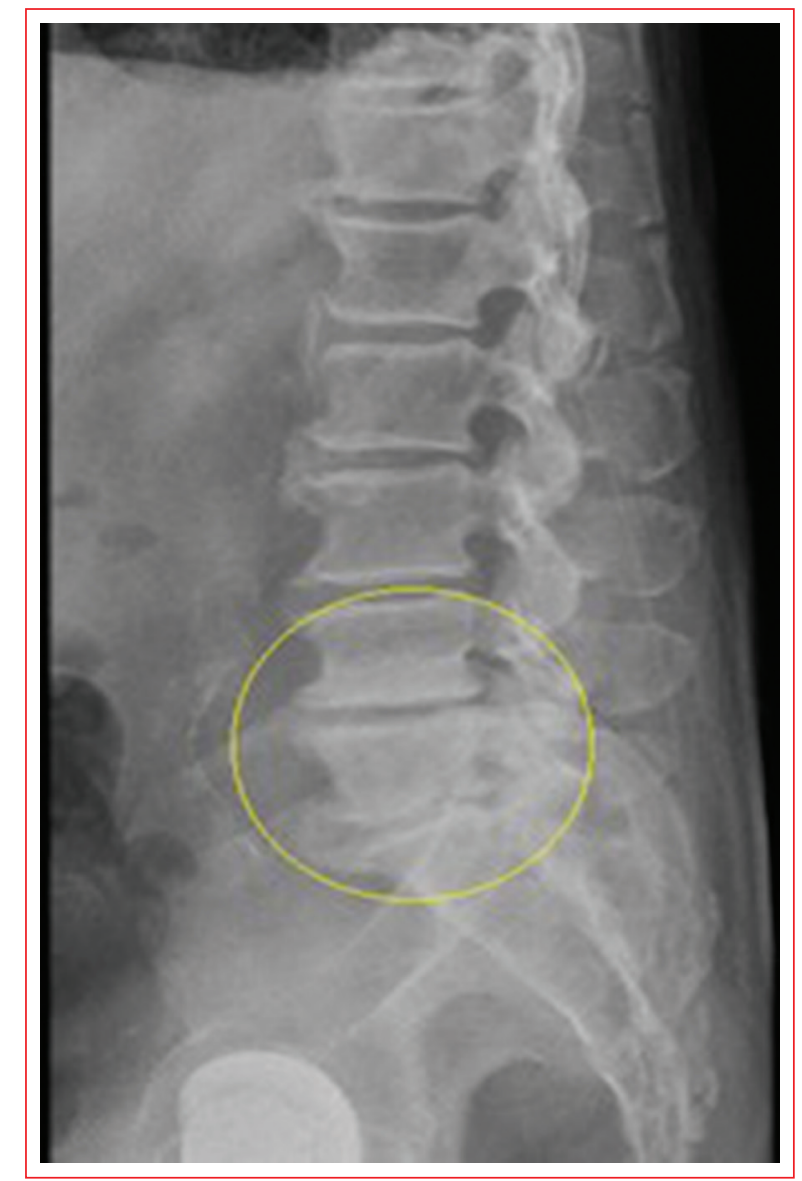

Figura 2. Espondilólisis con hiperostosis en L4-L5 y severos en L5-S1.

de estos, 146 con diseminación al componente óseo del esqueleto axial ${ }^{16}$.

En la infección por inoculación directa, los Staphylococcus coagulasa negativos y Propionibacterium acnes tienen mayor prevalencia, principalmente en pacientes con dispositivos de fijación ${ }^{14}$. Las infecciones polimicrobianas y multifocales son raras en menos del 10 y $5 \%$ respectivamente ${ }^{15}$.

\section{Diagnóstico}

La lumbalgia es el síntoma cardinal presentado en el $86 \%$ de los casos, asociado en un $60 \%$ con fiebre. La radiculopatía, debilidad, parálisis, disestesias y retención urinaria pueden hallarse hasta en el $34 \%$ de los pacientes ${ }^{17}$.

En los paraclínicos séricos, la elevación de reactantes de fase aguda como la proteína $C$ reactiva y velocidad de sedimentación globular tienen una sensibilidad del $94-100 \%{ }^{8}$. La leucocitosis y neutrófilos $>80 \%$ 
Tabla 1. Tratamiento antibiótico según directriz de la guía de la Infectious Diseases Society of America (IDSA) 2015

\begin{tabular}{|c|c|c|}
\hline & Primera elección & Segunda elección \\
\hline $\begin{array}{l}\text { Staphylococcus susceptible a la } \\
\text { oxacilina }\end{array}$ & $\begin{array}{l}\text { Cefazolina } 1-2 \mathrm{~g} \mathrm{IV} \text { cada } 8 \mathrm{~h}^{*} \\
\text { Ceftriaxona } 2 \mathrm{~g} \text { IV cada } 24 \mathrm{~h}^{*}\end{array}$ & $\begin{array}{l}\text { Vancomicina } 15-20 \mathrm{mg} / \mathrm{kg} \text { IV cada } 12 \mathrm{~h} \\
\text { Daptomicina } 6-8 \mathrm{mg} / \mathrm{kg} \mathrm{IV} \mathrm{cada} 24 \mathrm{~h}^{*} \\
\text { Linezolid } 600 \mathrm{mg} \mathrm{IV} \mathrm{cada} 12 \mathrm{~h} \\
\text { Levofloxacino } 500-750 \mathrm{mg} \text { V0 cada } 24 \mathrm{~h}+ \\
\text { rifampicina } 600 \mathrm{mg} \text { cada } 24 \mathrm{~h}^{*}\end{array}$ \\
\hline $\begin{array}{l}\text { Staphylococcus resistente a la } \\
\text { oxacilina } \\
\text { Enterococcus resistente a la } \\
\text { penicilina }\end{array}$ & Vancomicina $15-20 \mathrm{mg} / \mathrm{kg} \mathrm{IV} \mathrm{cada} 12 \mathrm{~h}$ & $\begin{array}{l}\text { Daptomicina } 6-8 \mathrm{mg} / \mathrm{kg} \mathrm{IV} \text { cada } 24 \mathrm{~h}^{*} \\
\text { Linezolid } 600 \mathrm{mg} \mathrm{IV} \mathrm{cada} 12 \mathrm{~h} \\
\text { Levofloxacino } 500-750 \mathrm{mg} \text { VO cada } 24 \mathrm{~h}+ \\
\text { rifampicina } 600 \mathrm{mg} \text { cada } 24 \mathrm{~h}^{*}\end{array}$ \\
\hline $\begin{array}{l}\text { Enterococcus susceptible a la } \\
\text { penicilina }\end{array}$ & $\begin{array}{l}\text { Penicilina G } 20-24 \text { millones de unidades IV cada } \\
24 h^{*} \\
\text { Ampicilina } 1,200 \text { mg cada } 24 h^{*}\end{array}$ & $\begin{array}{l}\text { Vancomicina } 15-20 \mathrm{mg} / \mathrm{kg} \text { IV cada } 12 \mathrm{~h} \\
\text { Daptomicina } 6-8 \mathrm{mg} / \mathrm{kg} / \mathrm{IV} \text { cada } 24 \mathrm{~h}^{*} \\
\text { Linezolid } 600 \mathrm{mg} \text { IV cada } 12 \mathrm{~h}\end{array}$ \\
\hline Pseudomonas aeruginosa & $\begin{array}{l}\text { Cefepima } 2 \mathrm{~g} \text { cada } 12 \mathrm{~h}^{*} \\
\text { Meropenem } 1 \mathrm{~g} \text { cada } 8 \mathrm{~h}^{*} \\
\text { Doripenem } 500 \mathrm{mg} \text { cada } 8 \mathrm{~h} \text { IV }\end{array}$ & $\begin{array}{l}\text { Ciprofloxacino } 750 \mathrm{mg} \text { cada } 12 \mathrm{~h}^{*} \\
\text { Aztreonam } 2 \mathrm{~g} \text { cada } 8 \mathrm{~h}\end{array}$ \\
\hline Enterobacterias & $\begin{array}{l}\text { Cefepima } 2 \mathrm{~g} \text { cada } 12 \mathrm{~h}^{*} \\
\text { Ertapenem } 1 \mathrm{~g} \text { cada } 24 \mathrm{~h}^{*}\end{array}$ & Ciprofloxacino $500-760 \mathrm{mg}$ V0 cada $12 \mathrm{~h}^{*}$ \\
\hline Streptococcus betahemolítico & $\begin{array}{l}\text { Penicilina G } 20-24 \text { millones de unidades IV cada } \\
24 \mathrm{~h} \\
\text { Ceftriaxona } 2 \text { g IV cada } 24 \mathrm{~h}^{*}\end{array}$ & Vancomicina $15-20 \mathrm{mg} / \mathrm{kg}$ IV cada $12 \mathrm{~h}$ \\
\hline Salmonella spp. & Ciprofloxacino 500 mg IV cada 12 h* & Ceftriaxona 2 g IV cada $24 \mathrm{~h}^{*}$ \\
\hline
\end{tabular}

*Duración del tratamiento: 6 semanas.

GR: gramos; IV: intravenoso; VO: tomar vía oral; C/: cada; HR: horas.

están presentes en el 39-64\% y ello se relaciona con infecciones por Staphylococcus ${ }^{14}$. La identificación del agente etiológico se puede lograr mediante hemocultivos, que son positivos en el $58 \%$ de los casos.

\section{Radiografía de columna}

Son anormales en el $89 \%$ de los casos y se requieren de 3-6 semanas desde el inicio de los síntomas para apreciar destrucción ósea ${ }^{8}$. La espondilólisis multisegmentaria e hiperostosis en L4-L5 y L5-S1 con amplitud normal en los otros espacios intervertebrales son hallazgos característicos (Fig. 2) ${ }^{18}$.

\section{Resonancia magnética nuclear contrastada}

Es el gold standard en pacientes con choque séptico con sensibilidad del $97 \%$ y especificidad del $93 \%{ }^{18}$. El edema es el hallazgo característico, donde no es posible distinguir los márgenes entre el espacio discal y la médula vertebral, en T1 hay mayor intensidad de la señal del disco y la médula adyacente afectada en T2, involucrando el espacio y dos cuerpos vertebrales con realce periférico de los tejidos por el medio de contraste $^{8}$.
La enfermedad puede progresar a la formación de abscesos epidurales, con una morbimortalidad relativamente alta, se observan masas hipointensas en T1 y $\mathrm{T} 2$ en el espacio epidural con congestión del plexo o de las venas basivertebrales, compresión del saco tecal o medular y restricción en la difusión ${ }^{18}$.

\section{Tomografía contrastada}

Es más sensible que la radiografía, con rendimiento similar a la resonancia magnética cuando se utiliza con contraste intravenoso, se realiza cuando la resonancia está contraindicada, no está disponible o es ambigua ${ }^{18}$.

\section{Gammagrafía}

Se utiliza solo en contraindicación o no disponibilidad de la resonancia magnética. Tiene sensibilidad y especificidad alta, el radioisótopo de elección es el difosfonato de tecnecio-metileno secuencial 99m y 67 gammagrafía con citrato de galio por la mayor captación a este nivel ${ }^{18}$.

\section{Tratamiento}

En pacientes con choque séptico y síntomas neurológicos progresivos, el antibiótico debe iniciarse 
temprano y de forma empírica. En los pacientes sin déficit neurológico y estables, se recomienda iniciar el tratamiento antimicrobiano una vez se identifique el microorganismo, evitando patrones de resistencia ${ }^{8}$.

La terapia debe dirigirse hacia el cubrimiento de $S$. aureus resistente a la meticilina, Streptococcus spp. y bacilos gramnegativos. La primera elección es van-

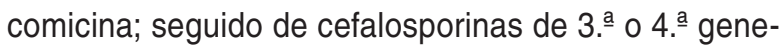
ración, y como tercera elección daptomicina o quinolonas en caso de reacciones adversas ${ }^{8,16}$ (Tabla 1).

La pobre vascularización ósea condiciona que la penetrancia antimicrobiana pueda tardar semanas, por esta razón se recomienda que el tratamiento sea prolongado por al menos seis semanas. El fracaso antibiótico está presente del 10-30\% facilitado en enfermedad multidisco, absceso epidural concomitante, infección por $S$. aureus y presencia de comorbilidades ${ }^{15}$ (Tabla 1).

Se recomienda el manejo quirúrgico en pacientes con septicemia, inestabilidad espinal con colapso vertebral $>50 \%$, absceso espinal $>2.5 \mathrm{~cm}$, déficit neurológico y deformidad progresiva, con el fin de reducir el volumen del tejido infectado, asegurar perfusión sanguínea y restaurar estabilidad espinal ${ }^{19}$.

\section{Conclusiones}

La espondilodiscitis es una entidad infrecuente, el síntoma cardinal es la lumbalgia asociada con fiebre. El antecedente de trauma o procedimientos a nivel axial suele orientar al diagnóstico, se puede confirmar con resonancia magnética de columna contrastada (gold standard), tomografía de columna contrastada o en su defecto gammagrafía como medio alternativo.

El principal agente etiológico es $S$. aureus, seguido de Streptococcus spp. y bacilos gramnegativos. En pacientes con sepsis o choque séptico se debe iniciar antibiótico de amplio espectro y la toma de hemocultivos para direccionar el tratamiento. Adicionalmente considerar el drenaje ante la presencia de abscesos, ya que se asocian con un peor pronóstico. Habitualmente se requieren cursos de antibióticos largos, aproximadamente de 60 días, producto de la pobre perfusión del disco vertebral que limita la penetrancia a estos tejidos.

\section{Financiamiento}

La presente investigación no ha recibido ninguna beca específica de agencias de los sectores públicos, comercial o sin ánimo de lucro.

\section{Conflicto de intereses}

Los autores declaran no tener conflicto de intereses.

\section{Responsabilidades éticas}

Protección de personas y animales. Los autores declaran que para esta investigación no se han realizado experimentos en seres humanos ni en animales.

Confidencialidad de los datos. Los autores declaran que han seguido los protocolos de su centro de trabajo sobre la publicación de datos de pacientes.

\section{Derecho a la privacidad y consentimiento infor-} mado. Los autores han obtenido el consentimiento informado de los pacientes y/o sujetos referidos en el artículo

\section{Bibliografía}

1. Maher C, Underwood M, Buchbinder R. Non-specific low back pain. Lancet. 2017;389:736-47.

2. Della-Giustina D. Evaluation and treatment of acute back pain in the Emergency Department. Emerg Med Clin North Am. 2015;33(2):311-26.

3. Maselli F, Palladino M, Barbari V, Storari L, Rossettini G, Testa M. The diagnostic value of Red Flags in thoracolumbar pain: a systematic review. DisabilRehabil. 2020;1-17.

4. Deyo R, Weinstein J. Low back pain. N Engl J Med. 2001;344(5):363-70.

5. Edlow J. Managing nontraumatic acute back pain. Ann Emerg Med. 2015;66(2):148-53

6. Márquez Sánchez P. Espondilodiscitis. Radiología. 2016;58:50-9.

7. Zimmerli W. Vertebral osteomyelitis. N Engl J Med. 2010;362(11):1022-9.

8. Cottle L, Riordan T. Infectious spondylodiscitis. J Infect. 2008;56(6):401-12.

9. IKouijzer I, Scheper H, de Rooy J, Bloem J, Janssen M, van den Hoven L, et al. The diagnostic value of 18F-FDG-PET/CT and MRI in suspected vertebral osteomyelitis a prospective study. Eur J Nucl Med Mol Imaging. 2017;45(5):798-805

10. Babic M, Simpfendorfer C. Infections of the spine. Infect Dis Clin North Am. 2017;31(2):279-97.

11. Principi N, Esposito S. Infectious discitis and spondylodiscitis in children. Int J Mol Sci. 2016;17(4):539.

12. Esendagli-Yilmaz G, Uluoglu O. Pathologic basis of pyogenic, nonpyogenic, and other spondylitis and discitis. Neuroimaging Clin N Am. 2015;25(2):159-61.

13. Giordan E, Marton E, Scotton G, Canova G. Outcomes and risk factors for spontaneous spondylodiscitis: Case series and meta-analysis of the literature. J Clin Neurosci. 2019;68:179-87.

14. Jensen AG, Espersen F, Skinhoj P, Rosdahl VT, Frimodt-Moller N. Increasing frequency of vertebral osteomyelitis following Staphylococ- cus aureus bacteraemia in Denmark 1980-1990. J Infect. 1997;34:113-8.

15. Berbari EF, Kanj SS, Kowalski TJ, Darouiche RO; Infectious Diseases Society of America. 2015 Infectious Diseases Society of America (IDSA) Clinical Practice Guidelines for the Diagnosis and Treatment of Native Vertebral Osteomyelitis in Adults. Clin Infect Dis. 2015;61(6):e26-46.

16. Mylona E, Samarkos M, Kakalou E, Fanourgiakis P, Skoutelis A. Pyogenic vertebral osteomyelitis: a systematic review of clinical characteristics. Semin Arthritis Rheum. 2009;39(1):10-7.

17. Diehn FE. Imaging of spine infection. Radiol Clin North Am. 2012;50(4):777-98.

18. Fragío Gil JJ, González Mazarío R, Salavert Lleti M, Román Ivorra JA Vertebral osteomyelitis; clinical, microbiological and radiological characteristics off 116 patientes. Med Clin (Barc). 2020;155(8):335-9.

19. Herren $C$, Jung N, Pishnamaz M, Breuninger M, Siewe J, Sobottke R Spondylodiscitis: Diagnosis and treatment options. Dtsch Arztebl Int. 2017;114(51-52):875-82. 